\title{
Need for a more developmental perspective: QTc prolongation under psychotropic medication
}

\author{
Veit Roessner $^{1} \cdot$ Nicole Wolff $^{1} \cdot$ Stefan $^{\text {Ehrlich }}{ }^{1} \cdot$ Robert Waltereit $^{1}$
}

Published online: 20 July 2017

(c) Springer-Verlag GmbH Germany 2017

There are several areas of child and adolescent psychiatry for which the quality and quantity of evidence are substantially lower than for psychiatric care of adults [1-4]. In this context, one of the most frequently asked questions is about the existence of appropriate and well-regulated pediatric psychopharmacological research to generate evidence as a basis for clinical recommendations [5]. Therefore, concerted efforts to facilitate pediatric psychopharmacological research are claimed again and again without noticeable progress probably because of the (still) limited economic perspectives for sponsors and the various ethical and other concerns and obstacles in investigating children and adolescents. But not only the "generation" of data on pediatric psychopharmacology, but also its "summary and critical evaluation" are still limited.

In our personal daily clinical experience in child and adolescent psychiatry, there are a handful of diagnostic and treatment recommendations that are based more on somewhat oversimplified statements from publications on adults than on comprehensive and well-balanced considerations of existing evidence including a thorough consideration of age group specificities.

The prime example for such a kind of "whisper down the lane" effect is the weighting of on the one hand sometimes urgent requirement of antipsychotic medication (e.g. acute psychotic episode) vs. on the other hand risk of QTc prolongation with the possible consequence of cardiovascular adverse events up to Torsades de Pointes (TdP). The latter can lead at worst to sudden cardiac death within minutes if

Veit Roessner

Veit.Roessner@uniklinikum-dresden.de

1 Department of Child and Adolescent Psychiatry, TU Dresden, Fetscherstrasse 74, 01307 Dresden, Germany not treated appropriately, but several authors suggest that the exact relationship between prolongation of the QTc interval and the risk of TdP remains elusive [6, 7]. Nevertheless, there seems to be a broad consensus that QTc interval > 450-500 ms, and/or QTc prolongation > $60 \mathrm{~ms}$ should be used as thresholds requiring increased attention in clinical settings. In addition, physicians should consider cardiovascular risk factors when choosing antipsychotic medications and monitor QTc intervals depending on patient and treatment-related risk factors, as is recommended by the German S3 and British NICE clinical guidelines for the treatment of adults with psychosis or schizophrenia [8, 9]. Notably, in both cases corresponding recommendations specific for children and adolescents do not exist.

There is some discussion as to the general validity of QTc prolongation in the individual patient due to ECG methodological issues (e.g. diurnal variation in the QTc interval) as well as its risk elevation of cardiovascular adverse events. While some authors argue that QTc prolongation is still the best clinical surrogate marker for TdP risk [10], some others criticize this as an oversimplified statement [11] and some even suggest newer surrogate markers (e.g. $\left.T_{\text {peak }}-T_{\text {end }}[12,13]\right)$ for ventricular arrhythmogenesis including drug-induced TdP. Furthermore, drug regulatory bodies and pharmaceutical companies have placed restrictions on some antipsychotic drugs which appear to have a low risk of TdP (for example, quetiapine). Conversely, other drugs with clear evidence of risk have the same level of restriction (for example, amisulpride) [6]. In this context, the definition and weighting of the (above mentioned) cardiovascular risk factors and the resulting effective individual risk for a cardiovascular adverse event are discussed controversially. Some admonitory authors call for a more detailed consideration of the risk of the 
individual patient for a cardiovascular adverse event, because such a weighting is the basis of treatment decisions. For example, Vieweg et al. [11] argue "...that the presence of individual-specific risk factors and the clinical milieu in which the 'culprit' drug is administered is unique to the particular instance, and such unique circumstances cannot be extrapolated to a general population-based analysis predicated on conventional statistical principles." Nevertheless, there are reports listing clinical scenarios that may indicate higher patient-related risk factors for QTc prolongation and/or cardiovascular adverse events of such a QTc prolongation, such as female sex, older age, electrolyte abnormalities (hypokalaemia, hypocalcaemia, hypomagnesaemia), cardiac ischaemia, cardiomyopathies, hypothyroidism, hypoglycaemia, prior ECG with a QTc $>450 \mathrm{~ms}$, palpitations at rest without anxiety, and dizziness or, especially, syncope upon exertion and family history of long QT syndrome or sudden death $[12,14]$. Also genetic factors account for a large amount of the variability in QTc as well as for congenital cardiac channelopathies including the Romano-Ward syndrome or the Jervell and LangeNielsen syndrome [15]. This may explain why some individuals are more predisposed to QTc prolongation and/or cardiovascular adverse events. But the most difficult issue is that QTc prolongation is indeed the most important risk factor for cardiovascular adverse events up to TdP, but also the established marker for TdP. Moreover, there is little information on what independently increases the risk of TdP for patients with QTc prolongation and on the complex interplay of all these identified risk factors. For example, amisulpride overdose commonly causes QTc prolongation but only a small proportion of cases with QTc prolongation actually develops TdP [16].

Although age- and sex-dependent normal values of the ECG are available [17, 18], the discussion about agedependency of the complex relationships among psychotropic medication, risk factors, and cardiovascular adverse events up to TdP development is limited. In this context, fortunately Jensen et al. [7] regretted in 2015 quite rightly that even reviews and clinical trials that focus on ECG changes explicitly in children and adolescents during antipsychotic treatment are scarce. In their opinion, this uncertainty has the consequence that clinicians may be unreasonably concerned when prescribing psychotropic medication. To overcome this problem, Jensen et al. [7] conducted the first systematic review and meta-analysis of QTc changes based on the clinical trials of antipsychotic treatment in this age group - a work that cannot be valued highly enough as an important step forward. Interestingly, their finding of the highest incidence of QTc prolongation for ziprasidone and very low/not different from pooled placebo for the other substances under investigation did not differ from similar analyses including exclusively or mainly adults.
At some points, Jensen et al. [7] linked their findings on QTc changes to risks for cardiovascular adverse events and discussed their clinical implications. They stated that the largest randomized active-controlled trial $(n=18,154$ adults) comparing olanzapine with ziprasidone revealed no difference in cardiac mortality or all-cause mortality over a 1-year period [19] calling into question the clinical relevance of QTc prolongation even with ziprasidone. This is in line with other statements based on clinical data; for example methadone is known to cause QTc prolongation but TdP occur only rarely and ceasing methadone is difficult because of its benefits and patient desire to continue. Also there was no relationship between methadone drug dose, QTc prolongation, and development of TdP [6, 20]. It should, however, be noted that results from "non-naturalistic" studies should be interpreted with caution, because participants in safety studies are usually free of many other, clinically encountered risk factors, and often a baseline QTc value exceeding $450 \mathrm{~ms}$ is an exclusion criterion [7].

In addition to the work of Jensen et al. [7], two recent studies contribute important findings for the specific situation of children and adolescents. Alda et al. [21] investigated over 12 months in a naturalistic, longitudinal multicenter study 216 children and adolescents receiving secondgeneration antipsychotic medications. Most patients were treated with risperidone, quetiapine or olanzapine. There were no significant QTc variations in the sample during follow-up. No patient had to discontinue psychotropic medication due to prolonged QTc. However, in this naturalistic study there was an important loss of participants to follow-up. Palanca-Maresca et al. [22] observed $n=101$ children and adolescents for months (range 1-39) treated with antipsychotic drugs, most of them risperidone or aripiprazole. Seven patients (6.9\%) had abnormal changes in QTc. No patient had a QTc interval of more than $500 \mathrm{~ms}$. All patients were clinically asymptomatic in cardiac terms. Taken together, these reports argue for a safe cardiac profile, especially of second-generation antipsychotics in children and adolescents.

In summary, recent reports indicate that in the vast majority of children and adolescents without the above-mentioned cardiovascular risk factors the risk of psychotropic medication-induced/associated cardiovascular adverse events presumably is very small. This applies even more for the risk of $\mathrm{TdP}$ - an already small risk in adults. Nevertheless, an ECG before starting psychotropic medication is recommended in current clinical practice, primarily due to the most prominent risk factor for a cardiovascular adverse event in this age group, i.e. elevated possibility of not yet recognized cardiac abnormalities. As an ECG before starting psychotropic medication is seen as a clinical standard, despite the poor evidence in children and adolescents, legal issues can be seen as a second reason. However, the somewhat divergent 
evaluation of and very limited base of evidence on the risk of psychotropic medication-induced/associated cardiovascular adverse events in children and adolescents stresses our plea: there is a strong need for pharmacologic research and clinical observational studies undertaken in the specific developmental age population of children and adolescents to improve decision certainty and to avoid the negative consequences of psychotropic non-treatment due to overcautious interpretation of pretreatment or follow-up ECG changes.

\section{References}

1. Gest S, Holtmann M, Bogen S et al (2016) Chronotherapeutic treatments for depression in youth. Eur Child Adolesc Psychiatry 25:151

2. Fonagy P, Speranza M, Luyten P et al (2015) ESCAP expert article: borderline personality disorder in adolescence: an expert research review with implications for clinical practice. Eur Child Adolesc Psychiatry 24:1307-1320. doi:10.1007/ s00787-015-0751-Z

3. Sharp C, Kalpakci A, Mellick W et al (2015) First evidence of a prospective relation between avoidance of internal states and borderline personality disorder features in adolescents. Eur Child Adolesc Psychiatry 24:283-290. doi:10.1007/s00787-014-0574-3

4. de Vries YA, de Jonge P, Kalverdijk L et al (2016) Poor guideline adherence in the initiation of antidepressant treatment in children and adolescents in the Netherlands: choice of antidepressant and dose. Eur Child Adolesc Psychiatry 25:1161

5. Schmäl C, Becker K, Berg R et al (2014) Pediatric psychopharmacological research in the post EU regulation 1901/2006 era. Z Kinder Jugendpsychiatr Psychother 42:441-449. doi:10.1024/1422-4917/a000322

6. Isbister GK (2015) Risk assessment of drug-induced QT prolongation. Aust Prescr 38:20-24. doi:10.18773/austprescr.2015.003

7. Jensen KG, Juul K, Fink-Jensen A et al (2015) Corrected QT changes during antipsychotic treatment of children and adolescents: a systematic review and meta-analysis of clinical trials. J Am Acad Child Adolesc Psychiatry 54:25-36. doi:10.1016/j. jaac.2014.10.002

8. Gaebel W (2005) Behandlungsleitlinie Schizophrenie. Springer Science \& Business Media, Berlin

9. National Collaborating Centre for Mental Health (Great Britain), National Institute for Clinical Excellence (Great Britain) (2002) Schizophrenia: core interventions in the treatment and management of schizophrenia in primary and secondary care. National Institute for Clinical Excellence
10. Nielsen J, Graff C, Kanters JK et al (2011) Assessing QT interval prolongation and its associated risks with antipsychotics. CNS Drugs 25:473-490. doi:10.2165/11587800-000000000-00000

11. Vieweg WVR, Hasnain M, Hancox JC et al (2013) Risperidone, QTc interval prolongation, and torsade de pointes: a systematic review of case reports. Psychopharmacology 228:515-524. doi:10.1007/s00213-013-3192-8

12. Panikkath R, Reinier K, Uy-Evanado A et al (2011) Prolonged Tpeak-to-tend interval on the resting ECG is associated with increased risk of sudden cardiac death. Circ Arrhythm Electrophysiol 4:441-447. doi:10.1161/CIRCEP.110.960658

13. Yamaguchi M, Shimizu M, Ino $\mathrm{H}$ et al (2003) $\mathrm{T}$ wave peak-to-end interval and QT dispersion in acquired long QT syndrome: a new index for arrhythmogenicity. Clin Sci Lond Engl 1979 105:671676. doi:10.1042/CS20030010

14. Correll CU, Lops JD, Figen V et al (2011) QT interval duration and dispersion in children and adolescents treated with ziprasidone. J Clin Psychiatry 72:854-860. doi:10.4088/ JCP.10m05990yel

15. Link MS (2017) Sudden cardiac death in the young: epidemiology and overview. Congenit Heart Dis. doi:10.1111/chd.12494

16. Isbister GK, Balit CR, Macleod D, Duffull SB (2010) Amisulpride overdose is frequently associated with QT prolongation and torsades de pointes. J Clin Psychopharmacol 30:391-395. doi:10.1097/JCP.0b013e3181e5c14c

17. Rijnbeek PR, Witsenburg M, Schrama E et al (2001) New normal limits for the paediatric electrocardiogram. Eur Heart J 22:702711. doi:10.1053/euhj.2000.2399

18. Rijnbeek PR, van Herpen G, Bots ML et al (2014) Normal values of the electrocardiogram for ages 16-90 years. J Electrocardiol 47:914-921. doi:10.1016/j.jelectrocard.2014.07.022

19. Strom BL, Eng SM, Faich G et al (2011) Comparative mortality associated with ziprasidone and olanzapine in real-world use among 18,154 patients with schizophrenia: the Ziprasidone Observational Study of Cardiac Outcomes (ZODIAC). Am J Psychiatry 168:193-201. doi:10.1176/appi.ajp.2010.08040484

20. Vieweg WVR, Hasnain M, Howland RH et al (2013) Methadone, QTc interval prolongation and torsade de pointes: case reports offer the best understanding of this problem. Ther Adv Psychopharmacol 3:219-232. doi:10.1177/2045125312469982

21. Alda JA, Munoz-Samons D, Tor J et al (2016) Absence of change in corrected QT interval in children and adolescents receiving antipsychotic treatment: a 12 month study. J Child Adolesc Psychopharmacol 26:449-457

22. Palanca-Maresca I, Ruiz-Antorán B, Centeno-Soto GA et al (2017) Prevalence and risk factors of prolonged corrected QT interval among children and adolescents treated with antipsychotic medications: a long-term follow-up in a real-World population. J Clin Psychopharmacol 37:78-83 